\title{
Spontaneous adverse event reporting by COVID-19 vaccinated healthcare professionals through an electronic form implemented by the hospital pharmacy
}

\author{
João Cruz ${ }^{1}$, Cristina Carvalho ${ }^{1}$, Paula Silva ${ }^{1}$, Leila Costa $^{1}$, Raquel Simões ${ }^{1}$, Rui Marques ${ }^{1}$, \\ Ana Castro $^{1}$, Ema Leite ${ }^{1}$, Luis Galaio ${ }^{1}$, Ana Correia ${ }^{1}$, and João Manuel Braz Gonçalves ${ }^{2}$ \\ ${ }^{1}$ Centro Hospitalar Universitário Lisboa Norte EPE \\ ${ }^{2}$ University of Lisbon Research Institute for Medicines and Pharmaceutical Sciences
}

September 24, 2021

\begin{abstract}
Aim Implementation of a web-form based pharmacovigilance plan for the spontaneous notification of adverse events to the Comirnaty $\mathbb{R}$ COVID-19 vaccine during its administration to hospital healthcare professionals. Methods An electronic pharmacovigilance form was developed containing 8 pre-defined event options, an open answer option for the description of other events and/or symptoms, and a question about the overall intensity of symptoms. The adverse events reports were standardised according to physiological and pathological condition. Results A total of 4119 adverse events notifications were obtained with a $45 \%$ rate of electronic notification. The most clinically relevant events reported were: tachycardia $(n=19)$, dyspnoea $(n=7)$, chest pain $(n=6)$, facial/labial oedema $(n=6)$, lipothymia $(n=5)$, bronchospasm $(n=2)$, herpetic infection $(n=2)$, vasculitis $(n=2)$, arrhythmia $(n=1)$, difficult to control arterial hypertension $(n=1)$, gastritis $(n=1)$ and spontaneous abortion $(n=1)$. Regarding the intensity of symptoms $(\mathrm{n}=2928), 70.0 \%$ were reported as mild, $25.8 \%$ as moderate and $4.27 \%$ as severe, with higher intensity in the 2nd dose compared to 1st dose. The highest frequency of severe events were reported in the groups from 40 to 59 years in both vaccination periods. During the vaccination process, no hospitalisations and no deaths were notified and/or recorded. Conclusion In this real world study, comparing with Comirnaty clinical trials program, it was observed a higher frequency of adenomegaly and gastrointestinal disorders. Noteworthy, the notification of a case of miscarriage. The use of hospital pharmacy pharmacovigilance electronic forms, seemed to be relevant to notification adherence and to obtain a greater and faster knowledge of COVID-19 vaccine safety profile.
\end{abstract}

\section{Hosted file}

Article Pharmacovigilance Comirnaty_BJCP.docx available at https://authorea.com/users/435222/ articles/538391-spontaneous-adverse-event-reporting-by-covid-19-vaccinated-healthcareprofessionals-through-an-electronic-form-implemented-by-the-hospital-pharmacy 


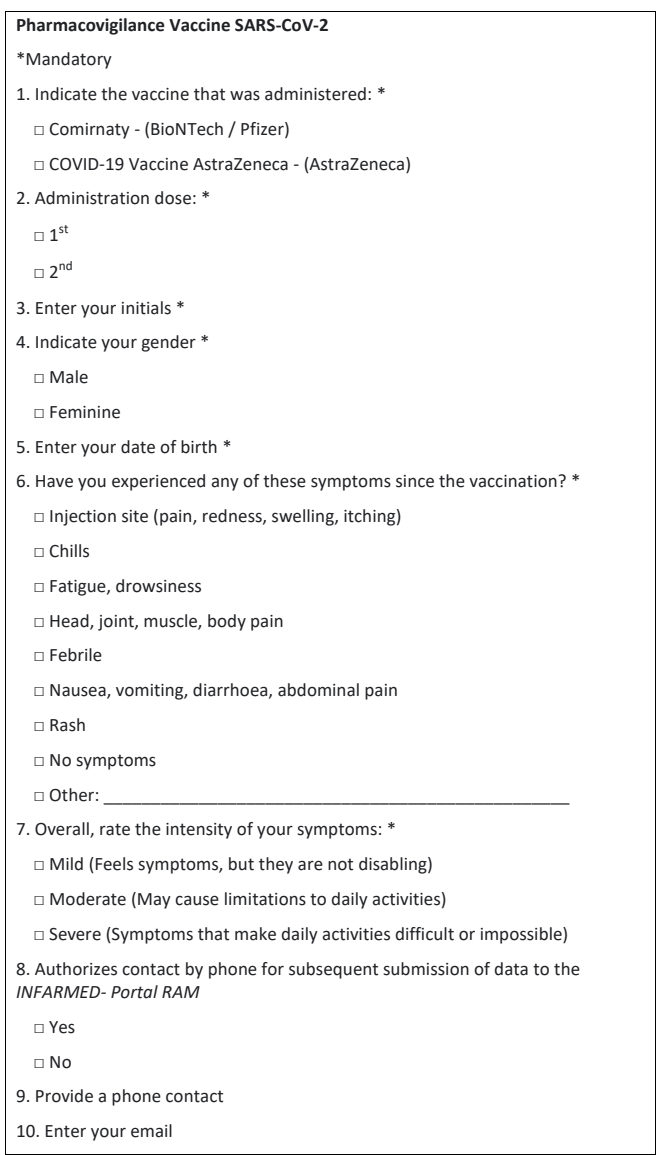




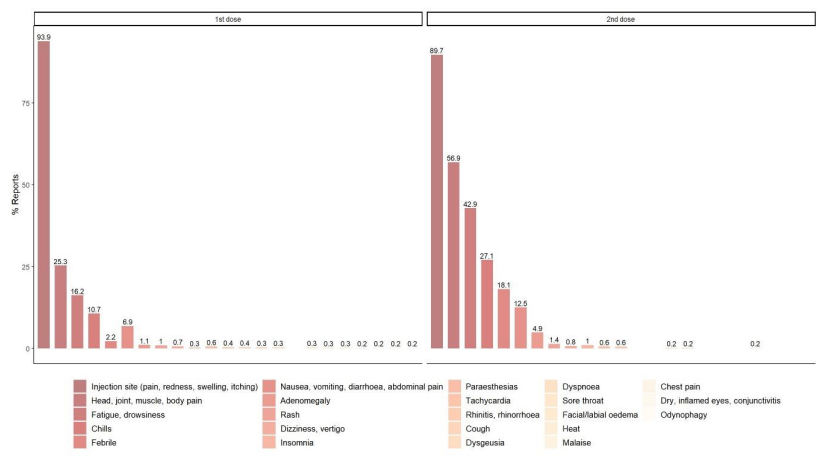




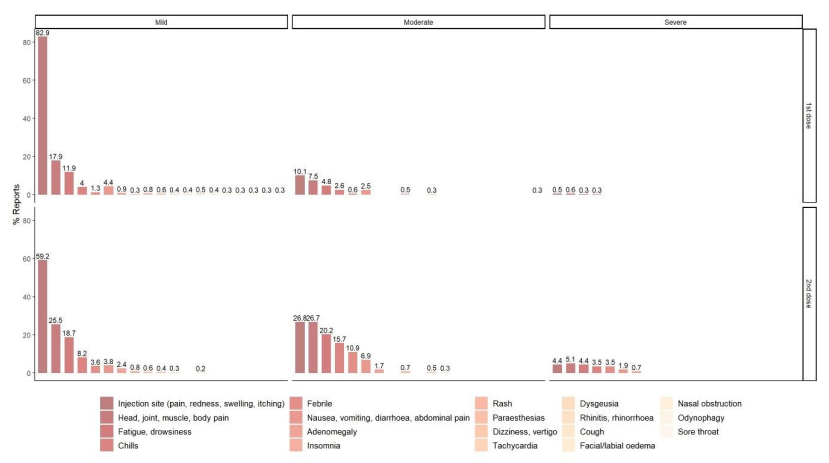

